\title{
Elevation of Nerve Growth Factor and Antisense Knockdown of TrkA Receptor during Contextual Memory Consolidation
}

\author{
Nancy J. Woolf, ${ }^{1}$ Amy M. Milov, ${ }^{1}$ Erik S. Schweitzer, ${ }^{2}$ and Ali Roghani ${ }^{3}$ \\ ${ }^{1}$ Department of Psychology and ${ }^{2 B}$ Brain Research Institute, University of California at Los Angeles, Los Angeles, California \\ 90095, and '3epartment of Pharmacology, Texas Tech University Health Sciences Center, Lubbock, Texas 79430
}

\begin{abstract}
We report here a series of experiments establishing a role for nerve growth factor and its high-affinity receptor TrkA in contextual memory consolidation. In all experiments, we trained rats in a novel chamber using tone and shock. Our first experiment revealed that endogenous nerve growth factor (NGF) increases in the hippocampus at a critical time during consolidation that occurs 1 week after training. NGF levels at other intervals ( $24 \mathrm{hr}$ and 2 and 4 weeks after training) did not differ from those of naive control animals. In our second experiment, we blocked effects that NGF has at 1 week after training by infusing antisense TrkA phosphorothioate DNA oligonucleotide. Reduction of septohippocampal TrkA receptor expression selectively impaired memory consolidation for context but not for tone. Animals with antisense TrkA oligonucleotide infused into the medial septal area or CA1 of the hippocampus froze less when placed in the training chamber than did animals infused
\end{abstract}

Neurotrophins participate in plastic processes in the brain, including those that accompany memory consolidation. Nerve growth factor (NGF) plays a role in memory and attention tasks that rely on the cholinergic septohippocampal pathway. NGF deprivation impairs spatial learning, for example, and causes cholinergic hypofunction in the medial septal area (Van Der Zee et al., 1995). Infusions of NGF facilitate spatial memory performance and restore cholinergic activity in aged and experimentally impaired animals (Janis et al., 1995; Fischer et al., 1996; Markowska et al., 1996). Endogenous NGF levels also correlate with an animal's capacity for spatial learning (Henriksson et al., 1992; Katoh-Semba and Kato, 1994).

A recent study further showed a positive relationship between NGF-induced enhancement of memory and increases in cholinergic markers and cholinergic cell body area (Gustilo et al., 1999). Hippocampal NGF and NGF mRNA levels also increase after animals are exposed to an enriched environment (Torasdotter et al., 1998; Pham et al., 1999b). Because enriched environments increase learning in a general way, one might expect to find specific increases in NGF after specific learning experiences.

We predicted that fluctuations in NGF accompanying memory consolidation of a spatial context might occur in the hippocampus slightly before structural changes. We found previously that signs of structural plasticity (i.e., cytoskeletal protein degradation) ap-

Received Sept. 15, 2000; revised Nov. 1, 2000; accepted Nov. 14, 2000.

This work was supported by National Institutes of Health Grant NS 35656 to A.R. We thank Shazia Iqbal for her contributions.

Correspondence should be addressed to Dr. Nancy J. Woolf, Department of Psychology, University of California, Los Angeles, CA 90095-1563. E-mail: nwoolf@ucla.edu.

Copyright (C) 2001 Society for Neuroscience $0270-6474 / 01 / 211047-09 \$ 15.00 / 0$ with inactive randomized oligonucleotide. At 4 weeks after training, antisense TrkA oligonucleotide had no effect on freezing. Third, we correlated levels of freezing with choline acetyltransferase (ChAT) and vesicular acetylcholine transporter (VAChT) immunohistochemistry. Antisense TrkA infused into CA1 of the hippocampus reduced cell body cross-sectional area for cholinergic cells in the medial septal area and decreased the density of hippocampal terminals labeled for ChAT and VAChT proteins. Cholinergic cell body measurements were significantly correlated with freezing. Taken together, these results indicate a role for nerve growth factor acting via the TrkA receptor on ChAT and VAChT proteins in contextual memory consolidation.

Key words: neurotrophins; Pavlovian conditioning; neural plasticity; hippocampus; DNA oligonucleotides; choline acetyltransferase; vesicular acetylcholine transporter pear in the hippocampus 2 weeks after animals receive training that produces contextual memory (Woolf et al., 1999). In the present study, we used the same contextual memory task that was used in our previous study and evaluated NGF levels at time intervals ranging from $24 \mathrm{hr}$ to 4 weeks after training.

Next, we tested whether NGF and expression of its high-affinity receptor TrkA are essential for consolidating memory of a spatial context. We infused antisense TrkA phosphorothioate DNA oligonucleotides into the medial septal area and into CA1 of the hippocampus. It has been established that antisense TrkA oligonucleotide reduces the synthesis of TrkA receptor protein (Taglialatela et al., 1996).

Choline acetyltransferase (ChAT) and vesicular acetylcholine transporter (VAChT) represent two known cholinergic markers (Woolf, 1991; Butcher et al., 1992; Bejanin et al., 1994; Erickson et al., 1994; Roghani et al., 1994). In cholinergic presynaptic terminals, ChAT synthesizes acetylcholine (ACh), and VAChT is responsible for transport of ACh into synaptic vesicles for regulated exocytotic release (Parsons et al., 1993). The VAChT gene is localized to the first intron of the ChAT gene, and the expression of these two genes is coregulated (Bejanin et al., 1994; Erickson et al., 1994; Berrard et al., 1995; Berse and Blusztajn, 1995; Usdin et al., 1995). Via the TrkA receptor, NGF is known to upregulate ChAT and VAChT (Tian et al., 1996; Takei et al., 1997).

To establish effects on cholinergic cells and their markers, we assessed ChAT and VAChT expressions after TrkA antisense infusion. We also evaluated correlations between behavioral responses and ChAT- and VAChT-immunoreactive cell body crosssectional area in the medial septal area and assessed terminal fields in the hippocampus. 
Table 1. Outline of experiment 1: NGF assays

\begin{tabular}{lll} 
Training & Time intervals until testing & $\begin{array}{l}\text { Time until } \\
\text { killing }\end{array}$ \\
\hline $2 \mathrm{~d}$ & $0(24 \mathrm{hr}), 1,2$, and 4 weeks after training & $4 \mathrm{hr}$ \\
$2 \mathrm{~d}$ & 1 week & $1 \mathrm{hr}$ \\
\hline
\end{tabular}

Table 2. Outline of experiment 2: antisense TrkA or randomized control oligonucleotide

\begin{tabular}{llcc} 
Training & Brain area & Time until surgery & Time until testing \\
\hline $2 \mathrm{~d}$ & $\begin{array}{c}\text { Medial septal } \\
\text { area }\end{array}$ & $\begin{array}{c}\text { 1 and } 4 \text { weeks after } \\
\text { training }\end{array}$ & 24 hr after surgery \\
$2 \mathrm{~d}$ & $\begin{array}{l}\text { CA1 of the } \\
\text { hippocam- }\end{array}$ & 1 week after training & 24 and $48 \mathrm{hr}$ after \\
& pus & & surgery \\
& & &
\end{tabular}

Parts of this paper have been published previously in abstract form (Milov et al., 1997; Woolf et al., 1998).

\section{MATERIALS AND METHODS}

Antisense oligonucleotides. We used two commercially available synthetic DNA oligonucleotides: antisense TrkA oligonucleotide and a randomized control oligonucleotide. Both oligonucleotides were fully phosphorothioated. Antisense oligonucleotides directed to TrkA and randomized controls have been designed and manufactured by Biognostik (Göttingen, Germany). The antisense TrkA sequence (5'AACTGTTGTTGTGTCC-3' ${ }^{\prime}$ corresponds to nucleotides $623-638$ of rat TrkA (Meakin et al., 1992). A random oligonucleotide (5'GTAAATTGACCAGGAG-3') was used as a control. We dissolved these oligonucleotides in a buffer containing $10 \mathrm{~mm}$ Tris-Cl, $\mathrm{pH} 7.2$, and $1 \mathrm{~mm}$ EDTA to a final concentration of $200 \mu \mathrm{M}$.

Animals. Seventy-two female Sprague Dawley rats were subjects in the present experiments. These animals were humanely treated according to both National Institutes of Health and University of California at Los Angeles (UCLA) guidelines. Three-month-old rats bred at UCLA (weight, 220-250 gm) were selected, at which time they were removed from group cages, placed in individual stainless steel cages, handled daily, and fed ad libitum. A 12/12 hr light/dark cycle was maintained, and the animals were trained and tested during the light cycle.

Behavioral training and testing. For experiment 1 , we randomly assigned rats $(n=20)$ to one of five conditions: $0(24 \mathrm{hr}), 1,2$, and 4 week training-testing interval groups or a naive control group (see Table 1). Training consisted of placing the animals in a novel conditioning chamber where they received four tone-shock pairings (a $30 \mathrm{sec}, 96 \mathrm{~dB}, 2 \mathrm{kHz}$ tone and a $1 \mathrm{sec}, 1 \mathrm{~mA}$ grid shock) with an intertrial interval of $10 \mathrm{~min}$. Training continued for 2 consecutive days, and the different groups of trained rats were then tested at $24 \mathrm{hr}$ to 4 weeks after $2 \mathrm{~d}$ of training (Table 1). One group of naive control rats was also tested. An observer blind to each animal's condition scored behavior from videotapes. Periods of immobility or freezing were measured during a $30 \mathrm{sec}$ period starting 2 min after the rats were placed in the chamber. Two minutes after the onset of the first observation period, the tone came on, and freezing was measured during the $30 \mathrm{sec}$ tone duration. Rats were killed $4 \mathrm{hr}$ after testing, when the effects of testing had worn off.

To test for interactions between testing and training, we assigned rats $(n=16)$ to one of four conditions: trained and tested, trained and untested, untrained and tested, or untrained and untested. Training and testing were the same as above, except that the interval between training and testing was held constant at 1 week. We killed these rats within $1 \mathrm{hr}$ after testing, when the effects of testing were in place.

In experiment 2 , rats $(n=36)$ were randomly assigned to receive randomized control oligonucleotide or antisense TrkA oligonucleotide at two intervals after training (see Table 2). First, we trained these animals as described above for experiment 1 . Next, the animals received surgical inf usions of oligonucleotide either 1 or 4 weeks after training. Oligonu- cleotides were infused into the medial septal area (1 and 4 week groups) or CA1 of the hippocampus (1 week group only). We tested and killed animals $24 \mathrm{hr}$ after infusion, except for one hippocampal group that was infused, tested, and killed $48 \mathrm{hr}$ later.

ELISA measurements for NGF. Deeply anesthetized rats (injected with $90 \mathrm{mg} / \mathrm{kg}$ Nembutal, i.p.) were decapitated by guillotine. We then rapidly dissected and saved the hippocampus from both sides of each brain. We also saved the parietotemporal cortex as a reference brain area. Next, brain samples were homogenized in an extraction buffer containing 100 mM Tris-Cl, pH 7.0, $400 \mathrm{~mm} \mathrm{NaCl}, 20 \mathrm{~mm} \mathrm{CaCl}_{2}, 1 \mathrm{~mm}$ PMSF, $4 \mathrm{~mm}$ EDTA, and $7 \mu \mathrm{g} / \mathrm{ml}$ aprotinin, and then the mixtures were spun at 40,000 $\mathrm{rpm}$ for $10 \mathrm{~min}$ at $4^{\circ} \mathrm{C}$. We used the sandwich enzyme immunoassay technique to determine NGF levels (anti-NGF and standards were purchased from Roche Molecular Biochemicals, Indianapolis, IN). The supernatants were collected, and $100 \mu \mathrm{l}$ of each sample was pipetted into a well of a 96-well microtiter plate (Immulon 2; Dynex, West Sussex, United Kingdom) precoated with primary anti-NGF. After an overnight incubation at $4^{\circ} \mathrm{C}$, these plates were rinsed, then secondary anti-NGF- $\beta$ galactosidase was applied, and the mixture was left overnight at $4^{\circ} \mathrm{C}$. Subsequently, the substrate chlorophenol- $\beta$-D-galactopyranoside in a buffer containing $100 \mathrm{~mm}$ HEPES, pH 7.0, $150 \mathrm{~mm} \mathrm{NaCl}, 2 \mathrm{~mm} \mathrm{MgCl}_{2}$, and $1 \%$ bovine serum albumin was applied for $20 \mathrm{~min}$. The microtiter plates were read on a V-Max plate reader (Molecular Devices, Sunnyvale, CA) equipped with a $575 \mathrm{~nm}$ filter.

Infusion of antisense oligonucleotides. To anesthetize the rats, we injected $65-70 \mathrm{mg} / \mathrm{kg}$ Ketamine, along with $8.6 \mathrm{mg} / \mathrm{kg}$ xylazine, administered intraperitoneally. We then prepared rats for surgery and placed them in the stereotaxic instrument. The medial septal area $(n=20)$ or CA1 of the hippocampus (see below) was targeted. Stereotaxic coordinates for the medial septal area (the medial septal nucleus and vertical diagonal band nucleus) were set at $0.5 \mathrm{~mm}$ anterior and $0.5 \mathrm{~mm}$ lateral to bregma and 6.0 and $7.5 \mathrm{~mm}$ ventral to the surface of the skull according to the atlas of Paxinos and Watson (1997). At each brain site, we infused $5 \mu \mathrm{l}$ of oligonucleotide with a Hamilton microliter syringe. The first injections we made were unilateral (two injection sites at two depths, totaling 10 $\mu l$ ), after which we made bilateral injections (four injection sites, totaling 20 $\mu \mathrm{l})$.

Because TrkA expression is maximally suppressed by antisense after $24 \mathrm{hr}$ (Taglialatela et al., 1996), we waited $24 \mathrm{hr}$ and then tested and immediately killed the animals. We tested for tone and context-related memory by measuring freezing as described for experiment 1 . After the testing session, animals were deeply anesthetized $(90 \mathrm{mg} / \mathrm{kg}$ Nembutal, i.p.) and transcardially perfused.

Additional animals $(n=16)$ had antisense TrkA oligonucleotide and randomized oligonucleotide infused into CA1 of the hippocampus. The stereotaxic coordinates used to approach the dorsal CA1 of the hippocampus were $4.5 \mathrm{~mm}$ posterior and $3.0 \mathrm{~mm}$ lateral to bregma and 3.0 $\mathrm{mm}$ ventral to the surface of the skull. We infused a $5 \mu$ l oligonucleotide sample into each side of the hippocampus. The first half of these animals were tested $24 \mathrm{hr}$ after surgery, and the second half were tested $48 \mathrm{hr}$ later. We waited $48 \mathrm{hr}$ in the latter case because we were interested in retrograde effects on the cell bodies of medial septal neurons. On the basis of studies using fluorescent retrograde tracers, it takes $48 \mathrm{hr}$ for medial septal neurons to retrogradely transport maximal quantities of materials deposited in the hippocampus (Woolf et al., 1984).

Immunohistochemistry and tissue analyses. We processed brain tissue from animals in experiment 2 for immunohistochemistry according to previously described protocols (Oh et al., 1991; Roghani et al., 1998). Briefly, animals were deeply anesthetized and transcardially perfused with $50 \mathrm{ml}$ of PBS followed by $250 \mathrm{ml}$ of $4 \%$ paraformaldehyde in $0.1 \mathrm{M}$ phosphate buffer containing $0.2 \%$ picric acid at $4^{\circ} \mathrm{C}$. We cut the brains at $40 \mu \mathrm{m}$ intervals through the rostrocaudal extent of the medial septal area in the frontal plane of Paxinos and Watson (1997). All tissue sections through the medial septal nucleus and vertical diagonal band nucleus were saved, starting with $1.6 \mathrm{~mm}$ anterior to bregma through $0.3 \mathrm{~mm}$ posterior to bregma (anatomical landmarks were used to determine anterior-posterior level). One-third of these tissue sections were immunostained for ChAT, one-third were immunostained for VAChT, and one-third were immunostained for TrkA. We also saved sections through the dorsal CA1 region of the hippocampus from animals receiving infusions in that site. Tissue sections from differently trained animals were nicked at a predetermined place on the cortex (for later identification) and then collected in the same vial and stained free-floating under identical conditions.

Cholinergic neurons are known to coexpress ChAT and VAChT 


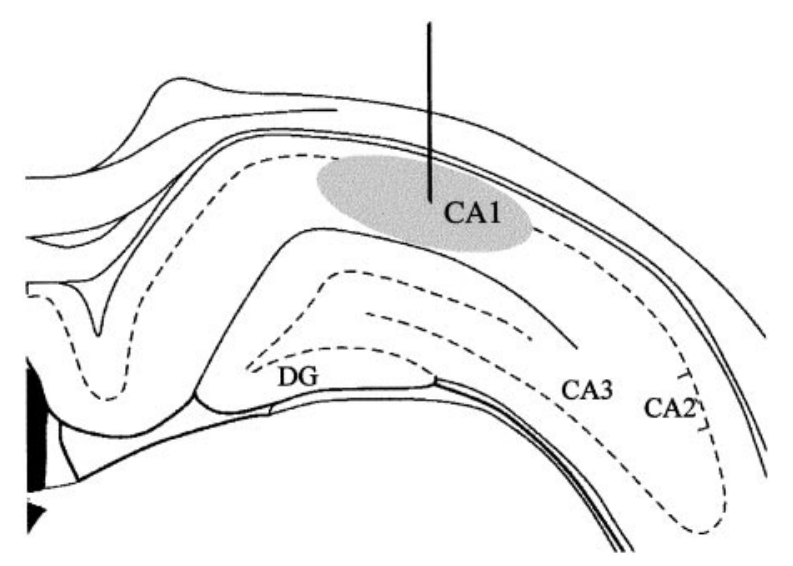

Figure 1. Schematic diagram of a typical infusion site of antisense TrkA oligonucleotide into $C A 1$ of the dorsal hippocampus. The extent was deduced from reduced immunohistochemical staining for ChAT and VAChT. The template is from Paxinos and Watson (1997). CA1, CA2, $C A 3$, Ammon's horn, regions $1-3 ; D G$, dentate gyrus.

proteins (Schafer et al., 1995; Gilmor et al., 1996; Arvidsson et al., 1997; Ichikawa et al., 1997; Roghani et al., 1998; Schäfer et al., 1998). We used antibodies directed against TrkA, ChAT, and VAChT to stain the cholinergic neurons of the medial septal area and the cholinergic terminals in the hippocampus. To exclude effects caused by nonspecific axonal damage, we also used antibodies to synaptophysin, which stain nerve terminals nonselectively, on hippocampal sections.

Anti-TrkA (polyclonal Ab 1577 purchased from Sigma, St. Louis, MO) was diluted $1: 500$ in PBS containing $0.1 \%$ sodium azide. Anti-ChAT (goat polyclonal $\mathrm{Ab}$ obtained from Chemicon, Temecula, CA) was diluted 1:200 in PBS with $0.1 \%$ sodium azide. Anti-VAChT (rabbit polyclonal Ab) (see Roghani et al., 1998) and anti-synaptophysin (monoclonal Ab, clone SVP-38, obtained from Sigma) were both diluted 1:1000 in PBS with $0.1 \%$ sodium azide. After $24 \mathrm{hr}$ of incubation at $4^{\circ} \mathrm{C}$ in the primary antibody, brain tissue sections were transferred to biotinylated secondary antibodies and then rinsed and exposed to a solution of avidin-biotin complex (Vector Laboratories, Burlingame, CA). Finally, tissue sections were incubated in a PBS solution containing $0.05 \%$ diaminobenzidine, $0.01 \%$ hydrogen peroxide, and $2.5 \%$ nickel ammonium sulfate.

We analyzed these tissue sections with an Olympus microscope equipped with a Bioquant image analysis system. We assessed infusion sites in the following ways. For animals inf used in the medial septal area, we first examined the tissue to establish the extent of protein disruption and then took low-power photomicrographs of the affected regions. We verified that the extent of protein disruption extended the same amount as did our preliminary injections of fluorescein (FITC)-conjugated antisense oligonucleotide provided by the manufacturer. For animals infused in CA1 of the hippocampus, we mapped the extent of the infusion on templates from the atlas of Paxinos and Watson (1997), as shown in Figure 1. We also took high-power photomicrographs of cholinergic terminal loss at the center of the infusion site.

An unbiased sampling (video-assisted) technique was used to count and measure cross-sectional areas of synaptophysin terminals in CA1 of the hippocampus. On the basis of anatomical landmarks, the region of CA1 located $4.5 \mathrm{~mm}$ posterior and $3.0 \mathrm{~mm}$ lateral to bregma was selected with the microscope set at $10 \times$. The lens was focused on the pyramidal cell layer (viewer assisted) and then moved into the immediately adjacent stratum radiatum (while the observer was not viewing the material under the microscope). The objective was then changed to $40 \times$, and computerassisted measurements were made. Slides from animals receiving different treatments were mixed together and assigned nondescriptive numbers before assessments were made such that the observer was blind to the training each animal received.

We also made analyses of remote effects in the medial septal area after infusions of oligonucleotides into CA1 of the hippocampus. Because ChAT and VAChT immunoreactivities completely fill cholinergic somata, we were able to evaluate cholinergic cell cross-sectional area in these immunostained sections. Cell body cross-sectional area measure-

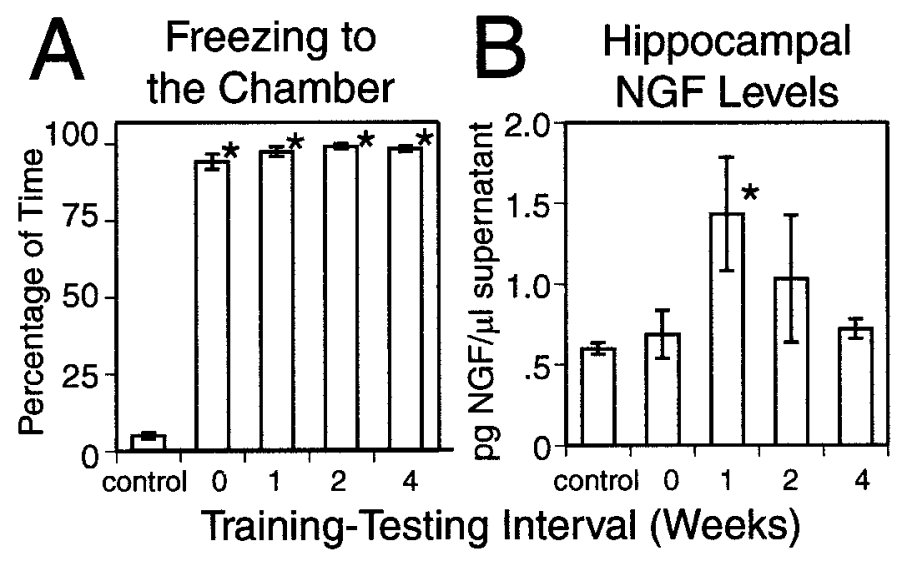

Figure 2. Behavioral responses at different training-testing intervals. $A$, The percentage of time animals were freezing (immobile except for respiratory movements) when placed in the training chamber. Values are for naive control animals and animals with $0(24 \mathrm{hr}), 1,2$, and 4 week training-testing intervals ( 4 animals in each group). Error bars show the SEM. Asterisks denote behavioral response levels that differed significantly from that of naive controls $(p<0.01)$. B, NGF levels in hippocampal homogenates collected from both sides of the brains of the naive control animals and the animals killed at 0 (24 hr), 1, 2, and 4 week intervals after training. Error bars show the SEM. At 1 week after training (see asterisk), NGF levels were significantly higher $(p<0.05)$ compared with that of naive control animals that were tested but not trained.

ments were made for every cholinergic neuron throughout the medial septal nucleus and vertical diagonal band nucleus in sections immunostained for ChAT or VAChT after digitization of the images onto a video screen. Cell fragments, identified by their irregular shape and small cross-sectional area, were not included in the analyses. The boundaries of these brain areas were taken from the atlas of Paxinos and Watson (1997). The observer was blind to the training each animal received. We attempted to use fully the stereological method to attain measurements that would enable us to estimate cell volume; however, we were cautious in doing so because we had introduced $5-10 \mu \mathrm{l}$ of fluid into each hemisphere, thereby altering the volume of affected brain areas. Nonetheless, we adhered to the principles of unbiased stereology in every other way possible (e.g., in tissue sectioning and selection of sections to analyze).

Statistics. We used a one-way ANOVA in most cases and a two-way ANOVA when specified. We also used the Student-Newman-Keuls post hoc test to assess differences in behavioral scores and NGF levels at different training-testing intervals and the Persson correlation coefficient to assess the significance of correlations between cell body cross-sectional area and behavioral responding.

\section{RESULTS}

\section{Time course of behavioral and hippocampal NGF responses}

The rats acquired a high rate of the behavioral response freezing after two training sessions of tone paired with shock delivered in a novel chamber. As shown in Figure $2 A$, the percentage of time animals froze was consistently high and significantly above levels shown by naive controls $(p<0.01)$. Without training, naive animals showed little or no freezing when placed in the chamber (see Fig. 2A, control bar).

Although the behavioral response was maintained across the 4 week period we assessed, hippocampal NGF levels showed a distinct pattern of change after training (see Fig. 2B). Animals killed $24 \mathrm{hr}$ after training ( 0 week) had NGF levels that were not significantly different from that of naive controls. A marked increase in NGF levels occurred, however, at 1 week after training. In these animals, NGF levels were $72 \%$ higher than those of 


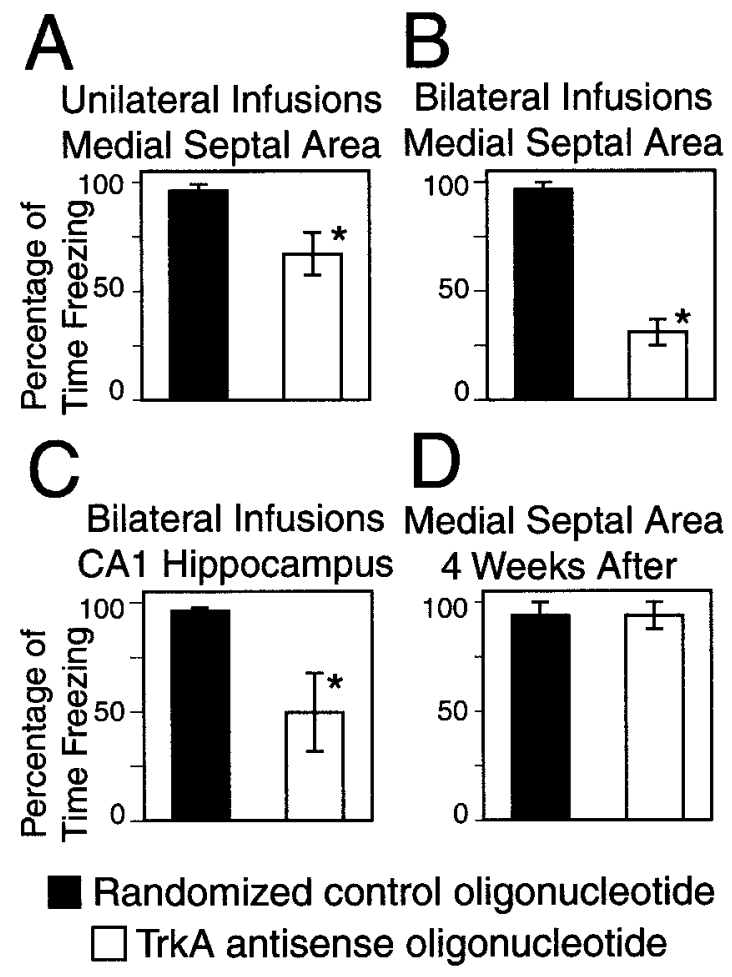

Figure 3. Behavioral outcomes of antisense oligonucleotide experiments. $A$, Freezing to the chamber is significantly decreased (asterisk, $p<$ 0.05 ) by unilateral infusions of antisense TrkA oligonucleotide into the medial septal area as compared with unilateral infusions of randomized control oligonucleotide. This was the outcome 1 week after training and $24 \mathrm{hr}$ after surgery. $B$, Freezing to the chamber is significantly decreased (asterisk, $p<0.01$ ) by bilateral infusions of antisense TrkA oligonucleotide into the medial septal area as compared with bilateral infusions of randomized control oligonucleotide. This was the outcome 1 week after training and $24 \mathrm{hr}$ after surgery. $C$, Freezing to the chamber is significantly decreased (asterisk, $p<0.05$ ) by bilateral infusions of antisense TrkA oligonucleotide into CA1 of the hippocampus as compared with bilateral infusions of randomized control oligonucleotide. This was the outcome 1 week after training and $48 \mathrm{hr}$ after surgery. $D$, Freezing to the chamber is not affected by bilateral infusions of antisense TrkA oligonucleotide into the medial septal area as compared with bilateral infusions of randomized control oligonucleotide 4 weeks after training and $24 \mathrm{hr}$ after surgery. Error bars in $A-D$ show the SEM; 3-4 animals served as subjects in each group of the above experiments.

naive controls, and this increase was significant $(p<0.05)$. NGF levels were not significantly elevated 2 weeks after training, and by 4 weeks after training, NGF levels in the hippocampus were indistinguishable from levels found in naive controls.

NGF quantitation in the parietotemporal cortex did not show the same pattern we found for hippocampal NGF. At 1 week after training, NGF in the parietotemporal cortex was found to decrease by $60 \%$ in trained animals as compared with naive controls $(p<0.05)$. This was the only time interval that revealed any differences between trained and control animals.

The effects of testing and the existence of any interactions between testing and training were also assessed by two-way ANOVA. To maximize effects of the test, rats were killed $1 \mathrm{hr}$ after testing. At this short interval after testing, the effect of testing was significant $(p<0.01)$. Training produced nonsignificant trends in the same direction as described above (i.e., hippocampal NGF levels of trained rats were increased $31 \%$ above those of untrained rats, and NGF in the parietotemporal cortex was decreased by $47 \%$ in trained rats compared with untrained rats). There were no significant interactions between effects of training and testing shown by our two-way ANOVA.

\section{Effects of antisense TrkA oligonucleotides on behavioral responses}

We placed antisense oligonucleotides into CA1 of the hippocampus to cause specific knockdown of cholinergic markers. This kind of specificity was sought, in part, because lesions of that structure have produced results that are open to varied interpretations (see Gewirtz et al., 2000). These infusions of antisense TrkA oligonucleotide produced changes in the behavioral responses that depended on the unilateral versus bilateral nature of the inf usion, the site, and time interval after training (see Fig. 3). Our first group of animals received unilateral infusions of antisense TrkA oligonucleotides into the medial septal area 1 week after training. As shown in Figure $3 A$, these animals froze significantly less when placed in the chamber compared with animals given randomized oligonucleotides $(p<0.05)$. As shown in Figure $3 B$, bilateral infusions of antisense TrkA oligonucleotide decreased freezing to an even greater extent than did unilateral infusions. The level of significance for freezing differences between bilateral infusion of antisense oligonucleotide and bilateral randomized oligonucleotide was greater $(p<0.01)$ than this comparison for unilateral infusions. This may reflect a doseresponse effect, because the bilateral infusions involved twice as much oligonucleotide as did the unilateral infusions (a total of 20 $\mu \mathrm{l}$ compared with $10 \mu \mathrm{l})$.

As shown in Figure $3 C$, antisense TrkA oligonucleotide also decreased freezing in animals receiving bilateral infusions into CA1 of the hippocampus compared with animals having randomized oligonucleotides infused into the same site $(p<0.05)$. When we used this infusion site, changes in freezing appeared to be caused by remote effects on the medial septal area because these alterations in behavior did not appear until $48 \mathrm{hr}$ after the infusions were made. Animals with infusions into CA1 of the hippocampus did not show contextual memory impairments at $24 \mathrm{hr}$ (data not shown), as did the animals with infusions into the medial septal area.

The sensitivity of the contextual memory to antisense TrkA oligonucleotides was time dependent. At 4 weeks after training, animals receiving infusions of antisense TrkA oligonucleotide into the medial septal area did not show decreased freezing compared with control animals given randomized oligonucleotide (Fig. 3D). Also, for all four groups of animals shown in Figure 3, freezing to tone was unaffected by the antisense TrkA oligonucleotide (data not shown). Hence the results of antisense TrkA oligonucleotide infusions into the hippocampus were specifically related to contextual memory consolidation.

\section{Histochemical confirmation of antisense TrkA knockdown near the infusion site and remote effects on medial septal neurons after hippocampal infusions}

Antisense TrkA oligonucleotide substantially decreased cholinergic markers along the septohippocampal pathway. Unilateral and, to a greater extent, bilateral infusions of antisense TrkA oligonucleotides decreased immunostaining intensity in the medial septal area (i.e., the medial septal nucleus and vertical diagonal band nucleus). As shown in Figure 4, the number of cells showing detectable amounts of ChAT, VAChT, or TrkA in animals receiving randomized control oligonucleotide $(A, C, E)$ was reduced by unilateral infusions of antisense TrkA oligonucleotide $(B, D, F)$. Approximately $35 \%$ of cells in the medial septal area 


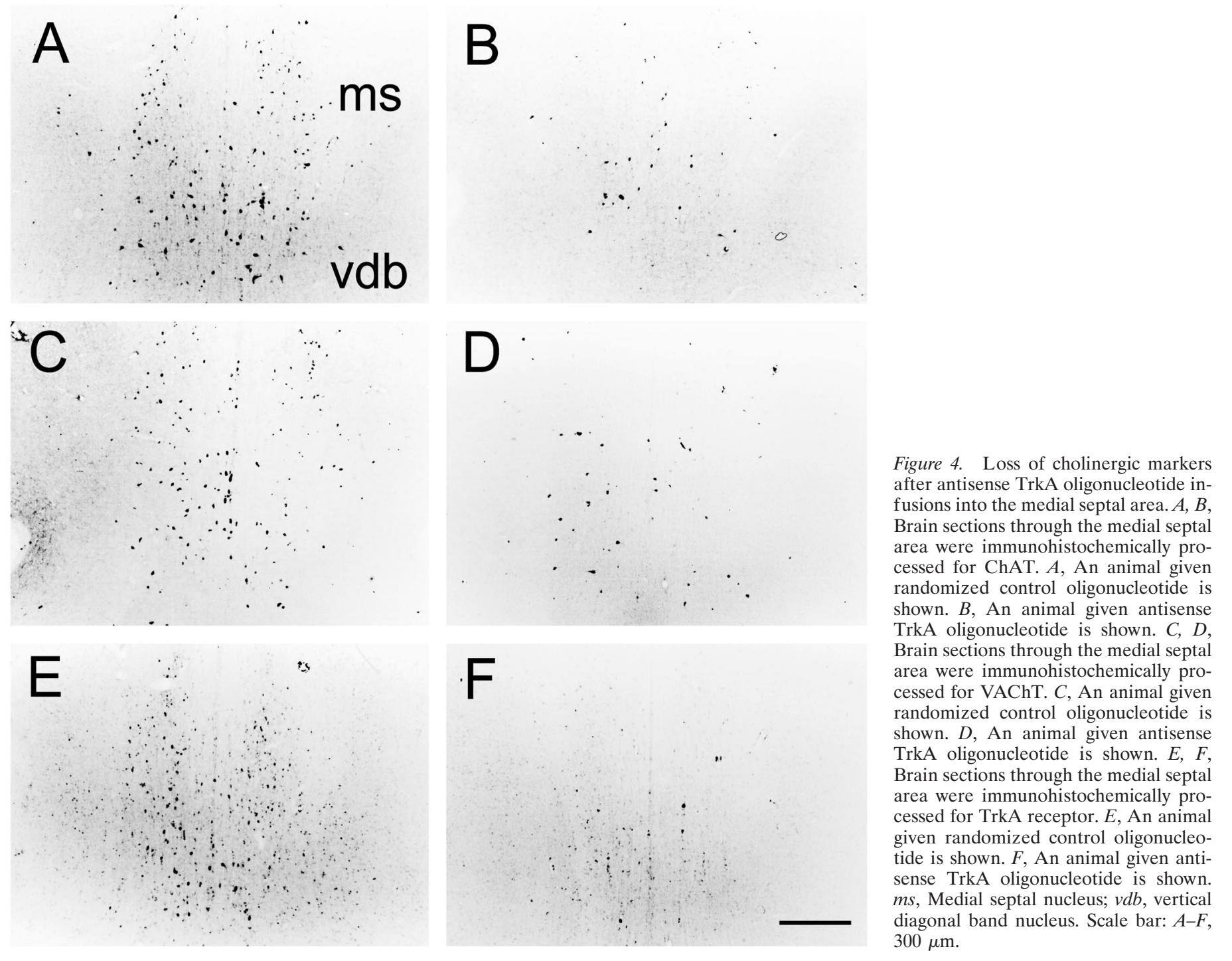

remained immunopositive for ChAT and VAChT, whereas only $20 \%$ remained immunopositive for TrkA, after unilateral infusions of antisense TrkA oligonucleotide as compared with randomized control oligonucleotide (Fig. 4, compare $B, D, F ; A, C, E$ ). Hence the strongest effect of the antisense TrkA oligonucleotide was directly on TrkA receptor expression. Bilateral infusions produced reductions of $>90 \%$ in the number of cells stained (data not shown).

Inf usions of antisense TrkA oligonucleotide into CA1 of the hippocampus caused local and remote effects. Near the infusion site, cholinergic terminal markers were reduced in density. As shown in Figure 5, the density and staining intensity of VAChT-containing terminals present in animals receiving randomized oligonucleotide $(A)$ were visibly reduced after infusions of antisense TrkA oligonucleotide $(B)$. The extent of reduced staining, as depicted in Figure 1, was limited to the CA1 region. The reduction in density and staining intensity was specific for the cholinergic markers VAChT (see above) and ChAT (data not shown) and was not observed in sections immunohistochemically processed with anti-synaptophysin, which stains all nerve terminals regardless of transmitter type (Fig. 5, compare $C, D$ ). Synaptophysin-stained terminals did not significantly differ between animals receiving TrkA oligonucleotide versus randomized oligonucleotide in terms of density $\left(6.8 \times 10^{4}\right.$ vs $5.6 \times 10^{4}$ terminals $/ \mathrm{mm}^{2}$ for the left hippocampus and $6.4 \times 10^{4}$ vs $5.3 \times 10^{4}$ terminals $/ \mathrm{mm}^{2}$ for the right hippocampus, respectively; $p>0.05$ ) or area of terminal boutons $\left(0.245\right.$ vs $0.258 \mu \mathrm{m}^{2}$ for the left hippocampus and 0.290 vs $0.273 \mu \mathrm{m}^{2}$ for the right hippocampus, respectively; $p>$ $0.05)$. Our observation that synaptophysin-stained terminals were not significantly different between groups of rats receiving different treatments possibly excludes any other reason (e.g., stage of estrous cycle) from conceivably accounting for significant differences between behavioral and cellular measurements between groups.

We also assessed the medial septal area for remote cellular changes after the infusions of antisense TrkA oligonucleotide into CA1 of the hippocampus. We further compared these remote changes, which were far removed from any damage at the infusion site, to the behavioral response rates. Although the entire hippocampus was not affected by our antisense TrkA infusions, cell bodies throughout the medial septal nucleus and the vertical diagonal band nucleus showed changes, suggesting that many of these cells retrogradely transported the antisense 
Figure 5. Terminals in CA1 of the hippocampus. VAChT-immunopositive terminals in CA1 of the hippocampus are intact after local infusions of randomized control oligonucleotide $(A)$ but are substantially reduced in the number in rats receiving antisense TrkA oligonucleotide (B). Synaptophysin-immunopositive terminals in CA1 of the hippocampus after local infusions of randomized control oligonucleotide $(C)$ or antisense TrkA oligonucleotide $(D)$ remain equally intact. Scale bar: $A-D, 20 \mu \mathrm{m}$.

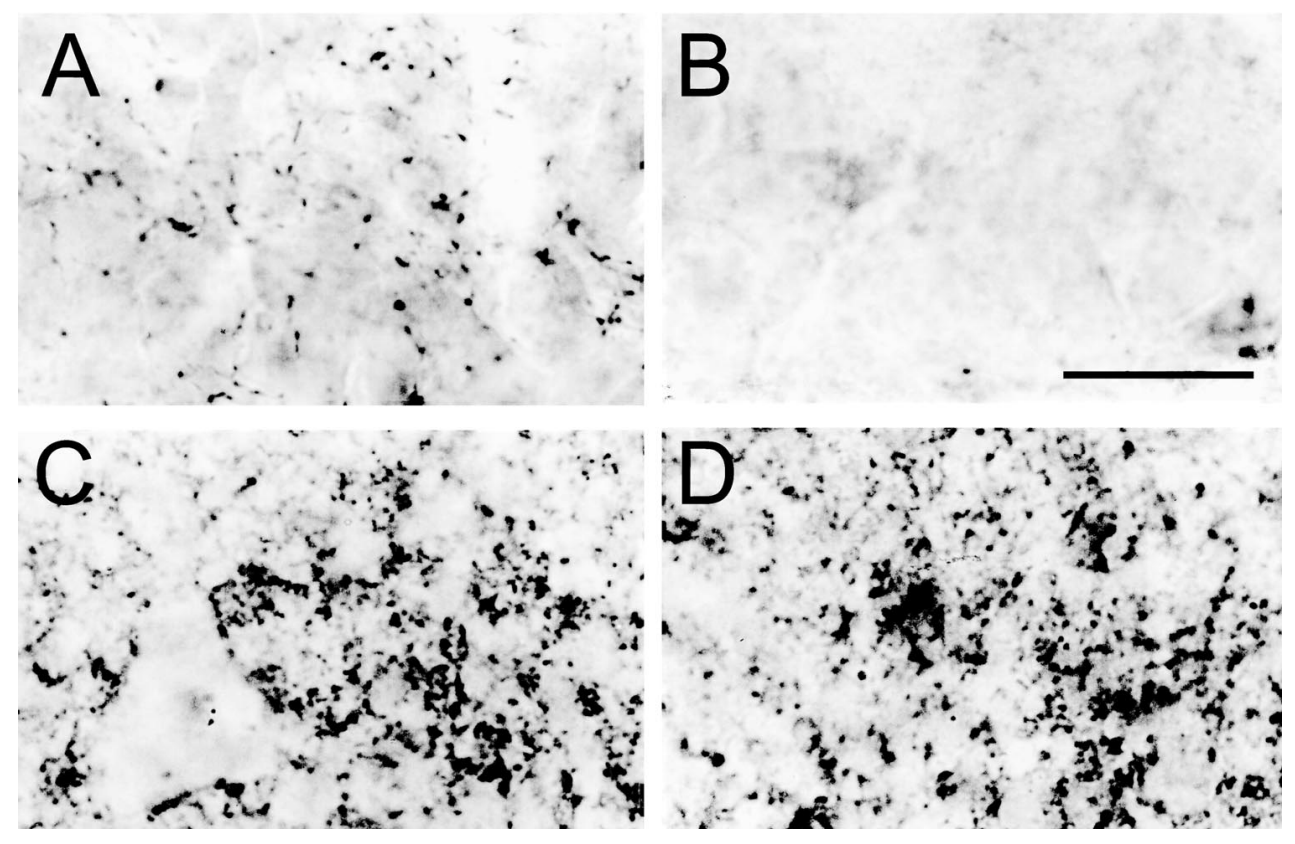

oligonucleotide from the CA1 region. As shown in Figure 6, the number of cells expressing ChAT in the medial septal nucleus and vertical diagonal band nucleus that were present after randomized oligonucleotide infusions $(A)$ was visibly reduced after infusions of antisense TrkA oligonucleotide $(B)$ into CA1 of the hippocampus. Moreover, the remaining ChAT-immunopositive cells had visibly reduced cell body cross-sectional areas (Fig. 6, compare $D, C$ ).

In our tissue samples, immunoreactive binding to the cytosolic proteins ChAT and VAChT was localized throughout the entire intracellular space; it filled each cell body completely, reaching the inner cell membrane and thereby enabling cell area measurements. Cell body cross-sectional area reductions were evident in analyses of both the ChAT- and the VAChTimmunostained sections. Pooling the data for animals receiving antisense TrkA oligonucleotide or randomized oligonucleotide, average cell body cross-sectional area for both ChATand VAChT-stained cells ranged from 129 to $181 \mu \mathrm{m}^{2}$, and these average cross-sectional areas varied across animals primarily in accordance with the treatment received (see Fig. 7). In both series of tissue sections, the average cell body crosssectional area for each animal correlated significantly with its behavioral response to the chamber. For ChAT cell body cross-sectional area and freezing, the correlation was more significant $(p<0.005)$ than that for VAChT cell body crosssectional area and freezing $(p<0.05)$.
Figure 6. Remote effects on cholinergic cells. ChAT-immunopositive cells in the medial septal area have unaltered cell bodies after local infusions of randomized control oligonucleotide $(A, C)$, but cells are visibly reduced in cross-sectional area after receiving antisense TrkA oligonucleotide $(B, D) . m s$, Medial septal nucleus; $v d b$, vertical diagonal band nucleus. Scale bar: $A, B, 400 \mu \mathrm{m}$ (low power); $C$, $D, 40 \mu \mathrm{m}$ (high power).
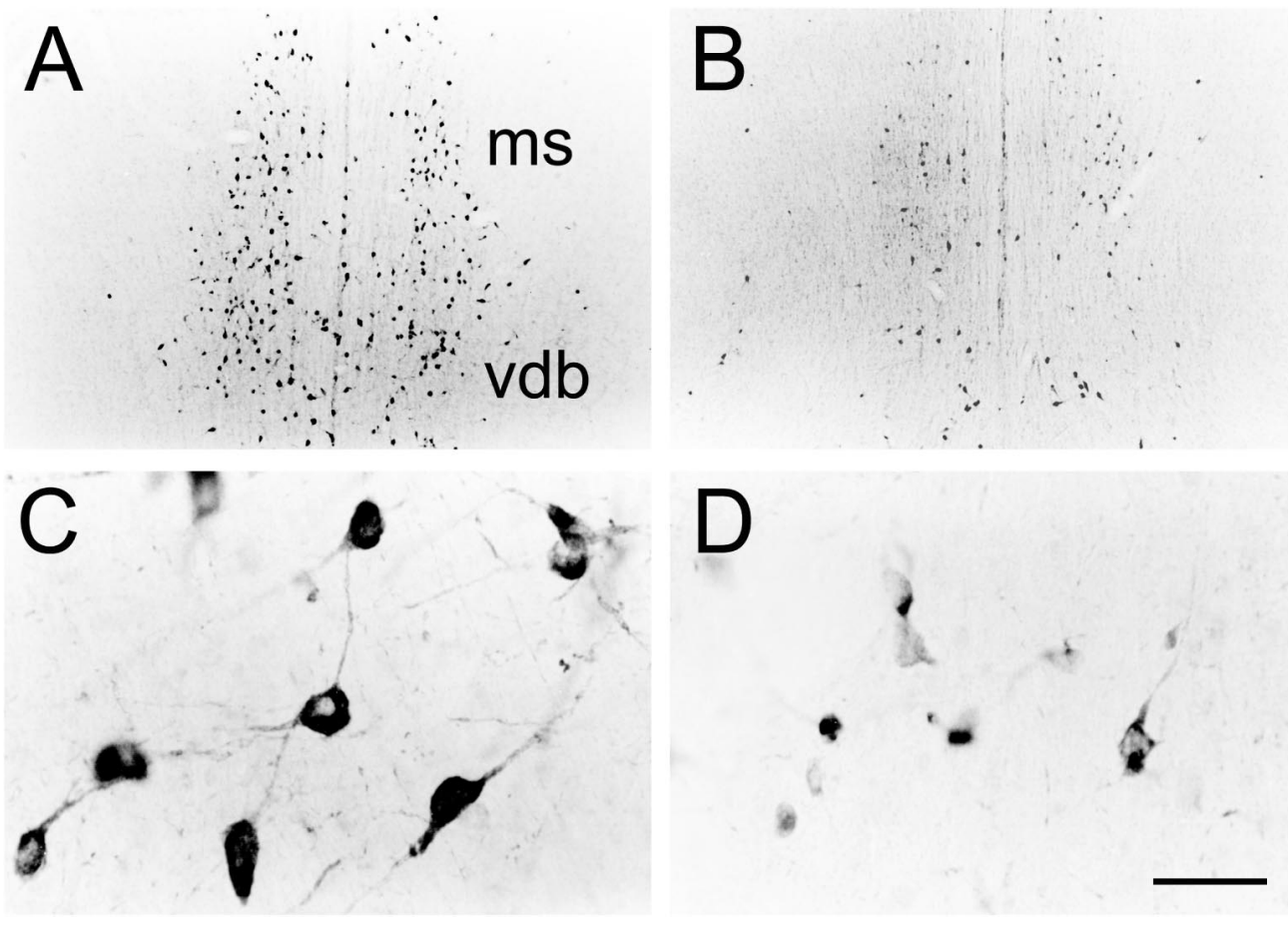


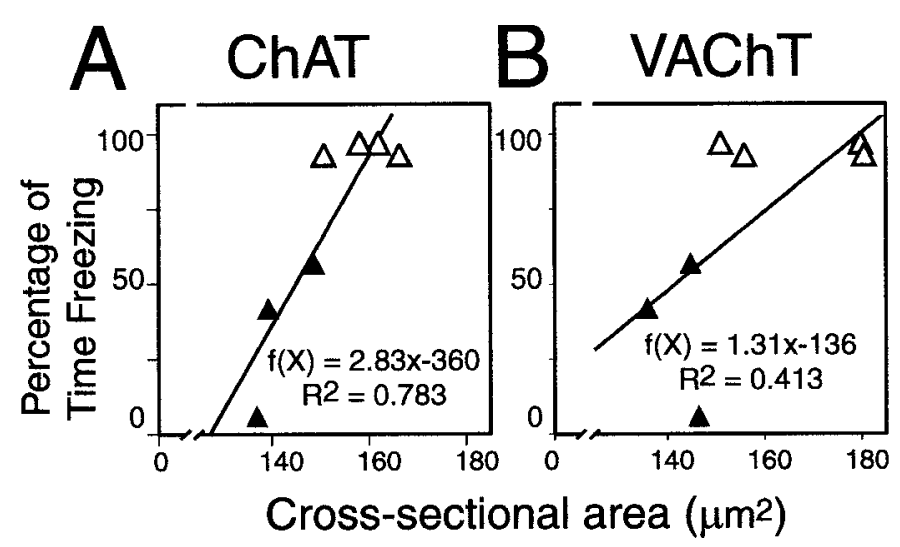

Figure 7. Graphs depicting correlations between cell body crosssectional area versus percentage of time freezing. A total of 1498 cells were measured. Black triangles represent the average cell body crosssectional area for animals receiving antisense TrkA oligonucleotide, and white triangles represent the average cell body cross-sectional area for animals receiving randomized control oligonucleotide. Linear plots illustrate the relationships between ChAT-immunopositive $(A)$ or VAChTimmunopositive $(B)$ cells and behavioral responses. Correlations were significant for both ChAT-immunopositive $(p<0.005)$ and VAChTimmunopositive $(p<0.05)$ cell body cross-sectional areas and percentage of time freezing.

\section{DISCUSSION}

\section{NGF alterations in the hippocampus at 1 week}

The results from our first experiment show that NGF is temporarily increased during a critical period of contextual memory consolidation. An increase in hippocampal NGF levels, above that of naive controls, was only apparent 1 week after training. There was no increase above control levels the day after training, nor was there any sign of an increase at 2 or 4 weeks after training. Thus, changes in NGF levels reflect a short-lived, sensitive period for plastic change in the hippocampus, a time during which the permanent memory for a new spatial context is stored.

Because NGF is a regulator of neural plasticity, this increase in NGF level might be expected to precede structural changes that accompany contextual memory consolidation, such as those involving the degradation of microtubule-associated protein-2 (MAP-2). Degradation of MAP-2 occurs in the hippocampus 2 weeks after the establishment of a conditioned fear response to the same spatial context (Woolf et al., 1999). Thus, the increased NGF level shown in the present study would come slightly before the MAP-2 degradation shown previously.

Initially, we were concerned that changes in NGF levels could be affected by mere exposure to the tone during testing. Simply removing rats from their home cages and exposing them to tone increases c-fos expression in many brain regions independent of conditioning (Smith et al., 1992). Induction of c-fos expression is maximal $1 \mathrm{hr}$ after tone stimulation (Zuschratter et al., 1995). Because c-fos protein is expressed in cells that respond to NGF (Sharp et al., 1993), increases in NGF level might be anticipated up to $1 \mathrm{hr}$ after testing. To avoid this problem, we waited until 4 $\mathrm{hr}$ after testing to assess for training-related differences in NGF level. No obvious effects of testing were observed, and because all groups of animals were similarly exposed to tone during testing, the specific NGF increase at 1 week could not be attributed to mere exposure to the tone or chamber during testing. In tissue assayed for NGF within $1 \mathrm{hr}$ after testing, the testing procedure, which consisted of exposure to the training chamber and the tone, produced a large effect. We found no interactions between training and testing in these animals, however, indicating that the test did not intensify or elicit any latent effects because of training in our first result. Hence we confidently interpret our NGF increase at 1 week to be wholly attributable to contextual memory consolidation occurring at this time. Our results are also consistent with enhanced retention of inhibitory avoidance behavior shown after NGF inf usions into CA1 of the hippocampus after training (Walz et al., 2000).

\section{TrkA knockdown decreases freezing responses elicited by the context}

Low levels of NGF precluded our NFG assay being limited to any subregion of the hippocampus; however, our antisense TrkA infusions were specific to CA1 of dorsal hippocampus. These TrkA knockdown experiments confirmed that increased hippocampal NGF present at 1 week after training is specifically involved with consolidation of contextual memory to the extent that freezing to context was impaired but freezing to tone was unimpaired. At 4 weeks after training, however, the contextual memory appeared to be fully consolidated, and antisense TrkA infusions into the medial septal area did not produce any deficit in freezing.

Although waiting 1 week after training produced the same behavioral deficit regardless of infusion site, the time after which surgical infusion caused behavioral deficits was site dependent. For the medial septal area, behavioral response was diminished $24 \mathrm{hr}$ after infusion. Antisense TrkA oligonucleotide inf used into CA1 hippocampus, however, produced effects on behavioral response after $48 \mathrm{hr}$ but not after $24 \mathrm{hr}$. This could be because extra time was needed for the antisense TrkA oligonucleotide to be retrogradely transported to the cell bodies of the cholinergic neurons, which are located in the medial septal area. After retrograde transport to medial septal cell bodies, one would expect reduced expression of cholinergic proteins and a behavioral deficit.

It is also worth mentioning that a very low level of TrkA mRNA has been detected in CA1 of the hippocampus by use of in situ hybridization with a sensitive $\mathrm{P}^{33}$-labeled riboprobe, and its presence has been confirmed using reverse transcription-PCR (Cellerino, 1996). Antisense TrkA oligonucleotide decreased the staining intensity of terminals in CA1 of the hippocampus in our experiment. This might represent a withdrawal of cholinergic terminals, which has been described previously for a TrkA antagonist (Debeir et al., 1999). A withdrawal of cholinergic terminals in the hippocampus might be expected to produce behavioral consequences. It is also possible that behavioral effects are mainly attributable to retrograde effects on medial septal neurons. This latter premise is supported by the time delay and the significant correlations between ChAT cell body cross-sectional area and behavioral response rate. It is also possible that local effects in CA1 of the hippocampus and remote effects in the medial septal area both contribute to diminished behavioral responses. Hence the antisense knockdown of the TrkA receptor could have inhibited two functionally important sites simultaneously.

\section{TrkA knockdown affects cholinergic markers ChAT and VAChT}

Our data suggest that NGF acted via the TrkA receptor to regulate cholinergic markers ChAT and VAChT during the sensitive period for contextual memory consolidation occurring 1 
week after training. We directly confirmed via immunohistochemical detection that the antisense TrkA oligonucleotide markedly decreased TrkA protein, as well as ChAT and VAChT proteins, in cholinergic cells of the medial septal area after inf usions into that site. Previous studies have suggested that NGF may participate in memory consolidation via the upregulation of ChAT (see introductory remarks). Accordingly, the increased hippocampal NGF we found during contextual memory formation would be expected to upregulate ChAT gene expression, as well as that of the closely linked VAChT gene. Moreover, synaptophysin immunoreactivity in hippocampal sections was unaltered, confirming that TrkA knockdown of ChAT and VAChT proteins, and not general axonal damage, was responsible for the memory deficit.

\section{Morphological and histochemical correlates of contextual memory}

A number of studies have reported changes in cholinergic cell body cross-sectional area that correlate with learning, memory, or exposure to an enriched environment. One of the initial reports was from our laboratory in which we reported increased cell body cross-sectional area after the acquisition of a conditioned emotion response to tone demonstrated by in situ hybridization for ChAT mRNA (Oh et al., 1996). Gustilo et al. (1999) reported recently that NGF increases cholinergic cell body area, and this increase may account for NGF-induced enhancement of recent and, to some extent, reference memory. Nonetheless, these authors importantly caution that corresponding effects on cholinergic terminals may be more critical to memory function than cell body alterations. Cell body area increases, however, have also been reported for TrkA- and p75-immunostained cholinergic cells in the medial septal area after a year-long exposure to an enriched environment (Pham et al., 1999a).

All the above studies reported increased cell body area under enhanced conditions. In this report, we present evidence that antisense to TrkA infused into CA1 of the hippocampus decreases the cell body cross-sectional area of medial septal neurons, and moreover, these cell cross-sectional areas are correlated with behavioral measures on the contextual memory task. The present data are highly compatible with previous results and warrant serious consideration of the basic tenant that not only do cholinergic cell bodies show increases in cross-sectional area during memory consolidation but these cross-sectional area increases are somehow necessary to the memory consolidation processes.

Although alterations in cell body area have now been repeatedly documented, effects on cholinergic terminals may be equally (or more) relevant to memory function. Cell body cross-sectional area alterations might signify, for example, reorganized trafficking to cholinergic terminals that will affect neurotransmitter release for extended periods of time. We were able to document dramatic local effects on VAChT terminals after antisense TrkA oligonucleotide was infused into CA1 of the hippocampus. It seems probable that cholinergic terminals are structurally altered shortly before the recipient cholinoceptive cells in the hippocampus undergo structural modification (see Woolf, 1996a,b, 1998). We plan to undertake more detailed studies relating the effects that antisense oligonucleotides have on cholinergic terminals in the hippocampus and correlative changes in behavioral response.

The present results demonstrating involvement of ChAT and VAChT proteins in the memory consolidation process may be interpreted as implying that changes in ACh synthesis and release are a part of this NGF-mediated process. In a previous study, NGF treatment of primary cultured rat embryonic septal cells enhanced expression of VAChT and ChAT proteins, as well as increased ACh synthesis and release (Oosawa et al., 1999). Thus, it is reasonable to assume that the memory impairment we observed in animals infused with the antisense TrkA oligonucleotide was caused, in part, by a reduction in synthesis and release of ACh by the presynaptic terminals in the CA1 of hippocampus.

In summary, these results support significant involvement of receptor-mediated mechanisms in conditioned behavior. Studying the consequences of such neuronal mechanisms should contribute to our understanding of the role of synaptic transmission by neurotransmitters, such as $\mathrm{ACh}$, in normal complex brain functions as well as in age-related neurological disorders.

\section{REFERENCES}

Arvidsson U, Riedl M, Elde R, Meister B (1997) Vesicular acetylcholine transporter (VAChT) protein: a novel and unique marker for cholinergic neurons in the central and peripheral nervous systems. J Comp Neurol 378:454-467.

Bejanin S, Cervini R, Mallet J, Berrard S (1994) A unique gene organization for two cholinergic markers, choline acetyltransferase and a putative vesicular transporter of acetylcholine. J Biol Chem 269:21944-21947.

Berrard S, Varoqui H, Cervini R, Israël M, Mallet J, Diebler MF (1995) Coregulation of two embedded gene products choline acetyltransferase and the vesicular acetylcholine transporter. J Neurochem 65:939-942.

Berse B, Blusztajn JK (1995) Coordinated up-regulation of choline acetyltransferase and vesicular acetylcholine transporter gene expression by the retinoic acid receptor alpha cAMP and leukemia inhibitory factor/ciliary neurotrophic factor signaling pathways in a murine septal cell line. J Biol Chem 270:22101-22104.

Butcher LL, Oh JD, Woolf NJ, Edwards RH, Roghani A (1992) Organization of central cholinergic neurons revealed by combined in situ hybridization histochemistry and choline- $O$-acetyltransferase immunocytochemistry. Neurochem Int 21:429-445.

Cellerino A (1996) Expression of messenger RNA coding for the nerve growth factor receptor trkA in the hippocampus of the adult rat. Neuroscience 70:613-616.

Debeir T, Saragovi HU, Cuello AC (1999) A nerve growth factor mimetic TrkA antagonist causes withdrawal of cortical cholinergic boutons in the adult rat. Proc Natl Acad Sci USA 96:4067-4072.

Erickson JD, Varoqui H, Schäfer MK, Modi W, Diebler MF, Weihe E, Rand J, Eiden LE, Bonner TI, Usdin TB (1994) Functional identification of a vesicular acetylcholine transporter and its expression from a "cholinergic" gene locus. J Biol Chem 269:21929-21932.

Fischer W, Sirevaag A, Wiegand SJ, Lindsay RM, Bjorklund A (1996) Reversal of spatial memory impairments in aged rats by nerve growth factor and neurotrophins 3 and $4 / 5$ but not by brain-derived neurotrophic factor. Proc Natl Acad Sci USA 91:8607-8611.

Gewirtz JC, McNish KA, Davis M (2000) Is the hippocampus necessary for contextual fear conditioning? Behav Brain Res 110:83-95.

Gilmor ML, Nash NR, Roghani A, Edwards RH, Yi H, Hersch SM, Levey AI (1996) Expression of the putative vesicular acetylcholine transporter in rat brain and localization in cholinergic synaptic vesicles. J Neurosci 16:2179-2190.

Gustilo MC, Markowska AL, Breckler SJ, Fleischman CA, Price DL, Koliatsos VE (1999) Evidence that nerve growth factor influences recent memory through structural changes in septohippocampal cholinergic neurons. J Comp Neurol 405:491-507.

Henriksson BG, Soderstrom S, Gower AJ, Ebendal T, Winblad B, Mohammed AH (1992) Hippocampal nerve growth factor levels are related to spatial learning ability in aged rats. Behav Brain Res 48:15-20.

Ichikawa T, Ajiki K, Matsuura J, Misawa H (1997) Localization of two cholinergic markers choline acetyltransferase and vesicular acetylcholine transporter in the central nervous system of the rat: in situ hybridization histochemistry and immunohistochemistry. J Chem Neuroanat 13:23-39.

Janis LS, Glasier MM, Martin G, Stackman RW, Walsh TJ, Stein DG (1995) A single intraseptal injection of nerve growth factor facilitates radial maze performance following damage to the medial septum in rats. Brain Res 679:99-109.

Katoh-Semba R, Kato K (1994) Age-related changes in levels of the beta-subunit of nerve growth factor in selected regions of the brain: comparison between senescence-accelerated SAM-P8 and senescenceresistant SAM-R1 mice. Neurosci Res 20:251-256.

Markowska AL, Price DL, Koliatsos VE (1996) Selective effects of nerve growth factor on spatial recent memory as assessed by a delayed 
nonmatching-to-position task in the water maze. $\mathrm{J}$ Neurosci 16:3541-3548.

Meakin SO, Suter U, Drinkwater CC, Welcher AA, Shooter EM (1992) The rat trk protooncogene product exhibits properties characteristic of the slow nerve growth factor receptor. Proc Natl Acad Sci USA 89:2374-2378.

Milov AM, Schweitzer ES, Woolf NJ (1997) NGF is transiently increased in hippocampus during consolidation of contextual memory. Soc Neurosci Abstr 23:221.

Oh JD, Butcher LL, Woolf NJ (1991) Thyroid hormone modulates the development of cholinergic terminal fields in the rat forebrain: relation to nerve growth factor receptor. Dev Brain Res 59:133-142.

Oh JD, Edwards RH, Woolf NJ (1996) Choline acetyltransferase mRNA plasticity with Pavlovian conditioning. Exp Neurol 140:95-99.

Oosawa H, Fujii T, Kawashima K (1999) Nerve growth factor increases the synthesis and release of acetylcholine and the expression of vesicular acetylcholine transporter in primary cultured rat embryonic septal cells. J Neurosci Res 57:381-387.

Parsons SM, Prior C, Marshall IG (1993) Acetylcholine transport, storage and release. Int Rev Neurobiol 35:279-393.

Paxinos G, Watson C (1997) The rat brain in stereotaxic coordinates. San Diego: Academic.

Pham TM, Ickes B, Albeck D, Söderström S, Granholm AC, Mohammed AH (1999a) Changes in brain nerve growth factor levels and nerve growth factor receptors in rats exposed to environmental enrichment for one year. Neuroscience 94:279-286.

Pham TM, Sèoderstrèom S, Winblad B, Mohammed AH (1999b) Effects of environmental enrichment on cognitive function and hippocampal NGF in the non-handled rats. Behav Brain Res 103:63-70.

Roghani A, Feldman J, Kohan SA, Shirzadi A, Gundersen CB, Brecha N, Edwards RH (1994) Molecular cloning of a putative vesicular transporter for acetylcholine. Proc Natl Acad Sci USA 91:10620-10624.

Roghani A, Shirzadi A, Butcher LL, Edwards RH (1998) Distribution of the vesicular transporter for acetylcholine in the rat central nervous system. Neuroscience 82:1195-1212.

Schafer MK, Weihe E, Erickson JD, Eiden LE (1995) Human and monkey cholinergic neurons visualized in paraffin-embedded tissues by immunoreactivity for VAChT the vesicular acetylcholine transporter. J Mol Neurosci 6:225-235.

Schäfer MK, Eiden LE, Weihe E (1998) Cholinergic neurons and terminal fields revealed by immunohistochemistry for the vesicular acetylcholine transporter. I. Central nervous system. Neuroscience 84:331-359.

Sharp FR, Sagar SM, Swanson RA (1993) Metabolic mapping with cellular resolution: c-fos vs 2-deoxyglucose. Crit Rev Neurobiol 7:5-28.

Smith MA, Banerjee S, Gold PW, Glowa J (1992) Induction of c-fos mRNA in rat brain by conditioned and unconditioned stressors. Brain Res 578:135-141.

Taglialatela G, Hibbert CJ, Hutton LA, Werrbach-Perez K, Perez-
Polo JR (1996) Suppression of p140TrkA does not abolish nerve growth factor-mediated rescue of serum-free PC12 cells. J Neurochem 66:1826-1835.

Takei N, Kuramoto H, Endo Y, Hatanaka H (1997) NGF and BDNF increase the immunoreactivity of vesicular acetylcholine transporter in cultured neurons from the embryonic rat septum. Neurosci Lett 226:207-209.

Tian X, Sun X, Suszkiw JB (1996) Developmental age-dependent upregulation of choline acetyltransferase and vesicular acetylcholine transporter mRNA expression in neonatal rat septum by nerve growth factor. Neurosci Lett 10:134-136.

Torasdotter M, Metsis M, Henriksson BG, Winblad B, Mohammed AH (1998) Environmental enrichment results in higher levels of nerve growth factor mRNA in the rat visual cortex and hippocampus. Behav Brain Res 93:83-90.

Usdin TB, Eiden LE, Bonner TI, Erickson JD (1995) Molecular biology of the vesicular ACh transporter. Trends Neurosci 18:218-224.

Van Der Zee CE, Lourenssen S, Stanisz J, Diamond J (1995) NGF deprivation of adult rat brain results in cholinergic hypofunction and selective impairments in spatial learning. Eur J Neurosci 7:160-168.

Walz R, Lenz G, Roesler R, Vianna MMR, Martins V, Brentani R, Rodnight R, Izquierdo I (2000) Time-dependent enhancement of inhibitory avoidance retention and MAPK activation by post-training infusion of nerve growth factor into CA1 region of hippocampus of adult rats. Eur J Neurosci 12:2185-2189.

Woolf NJ (1991) Cholinergic systems in mammalian brain and spinal cord. Prog Neurobiol 37:475-524.

Woolf NJ (1996a) Global and serial neurons form a hierarchically arranged interface proposed to underlie memory and cognition. Neuroscience 74:625-651.

Woolf NJ (1996b) The critical role of cholinergic basal forebrain neurons in morphological change and memory encoding: a hypothesis. Neurobiol Learn Mem 66:258-266.

Woolf NJ (1998) A structural basis for memory storage in mammals. Prog Neurobiol 55:59-77.

Woolf NJ, Eckenstein F, Butcher LL (1984) Cholinergic systems in the rat brain. I. Projections to the limbic telencephalon. Brain Res Bull $13: 751-784$

Woolf NJ, Roghani A, Milov AM (1998) Antisense DNA oligonucleotide probes to TrkA decrease contextual memory and cholinergic markers in septohippocampal neurons of rats. Soc Neurosci Abstr 24:1903.

Woolf NJ, Zinnerman MD, Johnson GVW (1999) Hippocampal microtubule-associated protein-2 alterations with contextual memory. Brain Res 821:241-249.

Zuschratter W, Gass P, Herdegen T, Scheich H (1995) Comparison of frequency-specific c-Fos expression and fluoro-2-deoxyglucose uptake in auditory cortex of gerbils (Meriones unguiculatus). Eur J Neurosci 7:614-626. 